\title{
Simultaneous synthesis of nanodiamonds and graphene via plasma enhanced chemical vapor deposition (MW PE-CVD) on copper
}

\author{
Steven Gottlieb ${ }^{1 *}$, Nicolas Wöhrl ${ }^{2}$, Stephan Schulz ${ }^{2}$ and Volker Buck ${ }^{1}$
}

*Correspondence:

steven.gottlieb@stud.uni-due.de

${ }^{1}$ Faculty of Physics and CENIDE, University Duisburg Essen,

Carl-Benz-Straße 199,

47057 Duisburg, Germany

Full list of author information is available at the end of the article

\begin{abstract}
The simultaneous growth of both nanodiamonds and graphene on copper samples is described for the first time. A PE-CVD process is used to synthesize graphene layers and nanodiamond clusters from a hydrogen/methane gas mixture as it is typically done successfully in thermal CVD processes for graphene synthesis. However, the standard thermal CVD process is not without problems since the deposition of graphene is affected by the evaporation of a notable amount of copper caused by the slow temperature increase typical for thermal CVD resulting in a long process time. In sharp contrast, the synthesis of graphene by PE-CVD can circumvent this problem by substantially shortening the process time at holding out the prospect of a lower substrate temperature. The reduced thermal load and the possibility to industrially scale-up the PE-CVD process makes it a very attractive alternative to the thermal CVD process with respect to the graphene production in the future. Nanodiamonds are synthesized in PE-CVD reactors for a long time because these processes offer a high degree of control over the film's nanostructure and simultaneously providing a significant high deposition rate. To model the co-deposition process, the three relevant macroscopic parameters (pressure, gas mixture and microwave power) are correlated with three relevant process properties (plasma ball size, substrate temperature and $\mathrm{C}_{2} / \mathrm{H}_{\mathrm{a}}$-ratio) and the influence on the quality of the deposited carbon allotropes is investigated. For the evaluation of the graphene as well as the nanodiamond quality, Raman spectroscopy used whereas the plasma properties are measured by optical methods. It is found that the diamond nucleation can be influenced by the $\mathrm{C}_{2} / \mathrm{H}_{\mathrm{a}}$-ratio in the plasma, while the graphene quality remains mostly unchanged by this parameter. Moreover it is derived from the experimental data that the direct plasma contact with the copper surface is beneficial for the nucleation of the diamond while the growth and quality of the graphene benefits from a larger distance to the plasma. Therefore, this work presents a basis for a method to tailor the deposition of graphene-diamond hybrid films using a MW PE-CVD process or to suppress the diamond deposition entirely if desired.
\end{abstract}

Keywords: Graphene, Nanodiamond, Hybrid, PE-CVD, Plasma, Raman, OES 


\section{Background}

Graphene as well as nanodiamonds, both allotropes of carbon, have obtained significant interest in the last couple of years from scientific as well as from industrial point of view due to their remarkable properties.

The regular hexagonal pattern in a crystalline monolayer of $\mathrm{sp}^{2}$-bonded carbon atoms is the reason for the remarkable mechanical, electronic, thermal, optical and chemical properties of graphene. Optical transparence of up to $97 \%$ and electron mobility above $15,000 \mathrm{~cm}^{2} / \mathrm{Vs}$ have been reported (Wang et al. 2010). The theoretical limits are estimated to be as high as $200,000 \mathrm{~cm}^{2} / \mathrm{Vs}$ for free-standing graphene (Akturk and Goldsman 2008) limited by the scattering of graphene's acoustic phonons. Due to these properties graphene receives much attention from fundamental as well as applied science and technology. However, the difference between theoretically possible values and the actually achieved ones originate from defects in the graphene sheet and interactions with the underlying substrate.

The first method to isolate graphene sheets was the method of mechanical exfoliation using Scotch tape to pull apart the layers of sample of highly oriented pyrolytic graphite and subsequently transfer single layers onto a $\mathrm{SiO}_{2}$ substrate (Novoselov et al. 2004). This method was also used by the Nobel price laureates K. S. Novoselov and A. K. Geim. As would seem natural, this approach is slow and the obtained graphene sheet is limited in size which results in the need for a production route suitable for industrial production.

One of the most frequently used methods to synthesize larger areas of high quality graphene is the use of CVD, in particular thermal CVD, which allows to deposit rather large areas with graphene of high quality (Reina et al. 2009). During the low-pressure CVD process, reactive gas species (mostly $\mathrm{H}_{2}$ and $\mathrm{CH}_{4}$ ) are fed into a hot-wall reactor at temperatures of around $1000{ }^{\circ} \mathrm{C}$ to initialize chemical reactions. Most of these CVD processes use copper substrates and take advantage of the catalytic influences of the copper on the dissociation of hydrogen ( $\mathrm{Li}$ et al. 2009; Vlassiouk et al. 2011). However, the high activation temperature for the catalytic reaction of methane causes significant evaporation of the substrate material even at temperatures far below the melting point of copper (Li et al. 2009) resulting in defects in the growing graphene monolayer. In combination with the slow increase of temperature in a typical thermal CVD reactor leading to a long process time, the process will be affected by the evaporation of a notable amount of copper.

Synthesis of graphene by PE-CVD is promising to reduce the problem of evaporation by substantially shortening the process time and having the lowering of the substrate temperature in prospect. The reduced thermal load and the possibility to industrially scale-up the PE-CVD process makes it a very attractive alternative to the thermal CVD process giving hope to synthesize a defect-free graphene layer on larger scale, which is essential for its industrial use such as energy applications (batteries, fuel cells) and semiconductor technology.

There have been some reports of plasma-based methods to decrease the process temperature including the use of microwave plasma CVD to synthesize graphene on nickel foil (Kim et al. 2011a), surface wave plasma CVD to synthesize graphene at temperatures in the range of $300-400{ }^{\circ} \mathrm{C}$ on large area conductive electrodes (Kim et al. 2011b; Kalita 
et al. 2012) and the plasma-assisted deposition of graphene on copper foils at temperatures down to $600{ }^{\circ} \mathrm{C}$ (Chan et al. 2013).

Recently a fast, versatile and reproducible PE-CVD method for the synthesis of SLG on large areas has been presented. It was shown that SLG sheets of several $\mathrm{mm}^{2}$ were deposited on copper substrates from the PE-CVD process (Woehrl et al. 2014).

However, during the PE-CVD process it was discovered that under certain process conditions a co-deposition of graphene and nanodiamond crystals could be observed on the copper surface.

Usually ultra-nanocrystalline diamond (UNCD) films with grain sizes between 5 and $100 \mathrm{~nm}$ are deposited from argon-rich $\mathrm{CH}_{4} / \mathrm{H}_{2}$ plasmas (Woehrl and Buck 2007; Buck and Woehrl 2008). In these processes it could be observed by the mean of plasma characterization (optical emission spectroscopy and mass spectroscopy) that by controlling the $\mathrm{C}_{2} / \mathrm{H}_{\alpha}$ ratio in the plasma it is possible to adjust the nucleation density and the growth rate of the deposited films. It was discovered that the nucleation density increases with the $\mathrm{C}_{2} / \mathrm{H}_{\alpha}$ intensity while the size of the individual diamond crystals decrease at the same time (Woehrl and Buck 2011).

In this work the influence of the plasma parameters (size of plasma ball, $\mathrm{C}_{2} / \mathrm{H}_{\alpha}$ ratio) on the growth process of these two carbon allotropes is investigated. It is aimed to control the deposition of graphene and nanodiamonds independently on each other. Special focus is on process parameters that enable the control of carbon phases formed on the surface to control the allotropes that are synthesized independently and to understand the processes that lead to the respective structures.

\section{Methods}

\section{Synthesis}

The co-deposition of Graphene and nanocrystalline diamond structures was performed with a $2.45 \mathrm{GHz}$ IPLAS CYRANNUS ${ }^{\circledR}$ I-6" plasma source with a maximum of $5 \mathrm{~kW}$ microwave power. The functional principle of this microwave plasma source is based on a resonator with annular slot antennas (CYRANNUS ${ }^{\circledR}$ 2001). This special setup allows the use of plasma from low pressure $\left(10^{-2} \mathrm{mbar}\right)$ to atmospheric pressure $(1 \mathrm{bar})$ and above.

The reaction chamber is a cylindrical shaped quartz tube with a diameter of $140 \mathrm{~mm}$ and a height of $140 \mathrm{~mm}$. The process gas is fed into the process chamber by a gas shower in the top flange and a molybdenum substrate holder of $100 \mathrm{~mm}$ diameter is mounted on the bottom flange (Fig. 1). The substrate is placed right below the plasma.

Carbon films were deposited on 50- $\mu$ m-thick copper foil (Puratronic $99.9999 \%$, item No.: 42972, Alfa Aesar GmbH \& Co KG, Karlsruhe, Germany) similar to those described elsewhere (Li et al. 2009).

After placing the copper foil in the center of the reaction chamber the reactor was evacuated to $1 \times 10^{-5}$ mbar. Hydrogen $(200 \mathrm{sccm})$ was introduced in the chamber and a plasma was ignited at a pressure of 5 mbar with a microwave power of 1-2 kW. Plasma cleaning was executed for 20 min to clean the substrate from all remaining organic contaminations and for chemical reduction of the surface. During that time the pressure was raised. Pressure was varied between 20 and 80 mbar for the different experiments. A suitable substrate temperature for the deposition process was measured with an optical 


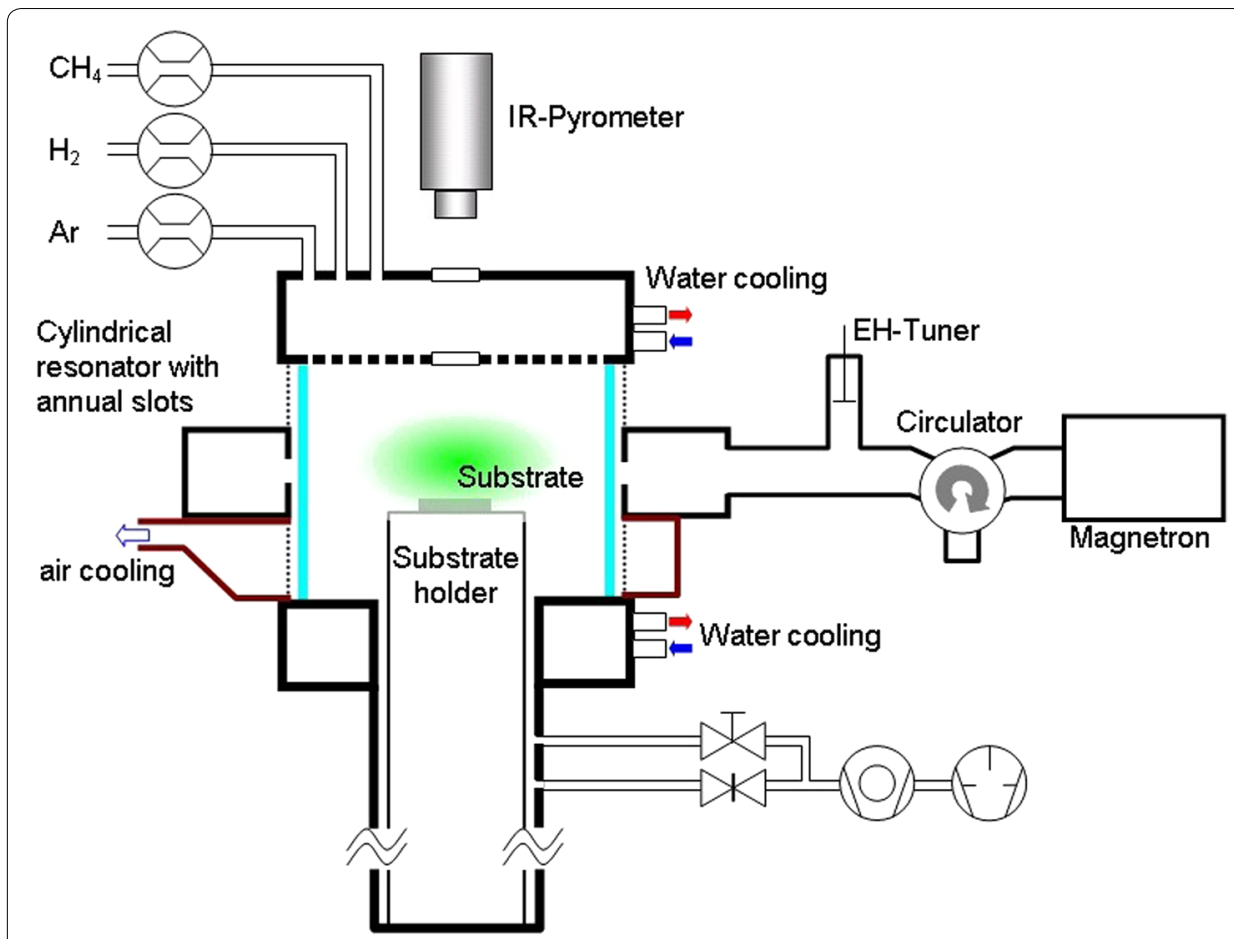

Fig. 1 Schematic diagram of the microwave plasma source and the substrate holder showing gas inlet in top left corner. Due to the microwaves coupled into the resonator provided with annular slots, a plasma is ignited

pyrometer to be $950-1030{ }^{\circ} \mathrm{C}$. The synthesis of carbon films starts with the introduction of 1.2-36 sccm methane into the process chamber and was performed for $8 \mathrm{~min}$ with the methane-hydrogen plasma at constant pressure and substrate temperature. The total gas flux (i.e. hydrogen + methane) was $120 \mathrm{sccm}$ during all coating processes. After that time the reactor was immediately evacuated to inhibit the etching influence of hydrogen on carbon structures at high temperatures. An overview of the process parameters can be found in Table 1.

The PE-CVD process uses hydrogen and methane as reaction gases as is typically done in thermal CVD process for the deposition of Graphene as well as diamond. The chemical fundamentals of the synthesis of Graphene are based on a physisorption process of hydrogen and a consecutive chemisorption process of methane (Zhang et al. 2011). Physically adsorbed $\mathrm{H}_{2}$ molecules decompose due to the high temperature on the copper surface. Consecutively chemisorption of methane molecules results in carbon species bonded to the top layered copper atoms, which become successively dehydrogenized and eventually building the graphene's honeycomb pattern. Due to the catalytic

Table 1 Range of relevant process parameters

\begin{tabular}{|c|c|c|c|c|c|c|c|}
\hline \multirow[t]{2}{*}{ Pressure } & \multirow[t]{2}{*}{ Power } & \multicolumn{3}{|c|}{ Cleaning process } & \multicolumn{3}{|c|}{ Synthesis } \\
\hline & & Time & $\mathrm{H}_{2}$ flow & $\mathrm{CH}_{4}$ flow & Time & $\mathrm{H}_{2}$ flow & $\mathrm{CH}_{4}$ flow \\
\hline 50-65 mbar & $1 \mathrm{~kW}$ & $20 \mathrm{~min}$ & $200 \mathrm{sccm}$ & - & $8 \mathrm{~min}$ & $118.8-84 \mathrm{sccm}$ & $1.2-36 \mathrm{sccm}$ \\
\hline
\end{tabular}

Relevant process parameters and the maximum and minimum values are depicted. While the cleaning process has been held constant as well as the process time, different microwave powers, process pressures and gas flows have been applied. The total gas flow, however, has not been alternated and has been kept at a constant value of $120 \mathrm{sccm}$ during the process 
influence of copper and the minor solubility of carbon in copper, this leads to the growth of monolayer graphene. The graphene growth process is self-limiting and therefore basically stops after the growth of a monolayer (Li et al. 2009).

As for the synthesis of diamond it has been well established that the methyl radical is the growth precursor for CVD-diamond growth. In standard hydrogen-rich gas compositions it is produced by the abstraction of one hydrogen atom from the methane molecule via its reaction with atomic hydrogen: $\mathrm{CH}_{4}+\mathrm{H} \rightarrow \mathrm{CH}_{3}+\mathrm{H}_{2}$. In a hydrogendeficient atmosphere-as used for UNCD growth-it is produced by electron collisional impact. This contribution leads to a non-vanishing growth rate even in hydrogen-free gas mixtures (Gruen 1999). Thus, it follows that-at constant gas composition, gas pressure, microwavepower, and substrate temperature-the plasma chemistry is fixed and the growth rate of diamond grains should be constant. There is evidence that the nucleation process for diamond differs from these reactions (Buck 2008). Since in deposition of nanocrystalline diamond films the strongest visible emission in the optical spectrum comes from the $\mathrm{C}_{2}$ line the $\mathrm{C}_{2}$ dimer was viewed as main growth and nucleation species (Gruen 1999; Gruen et al. 1994). Recently it was resolved for the first time by separate examination of the nucleation and the growth of diamond that both are consecutive but yet independent growth steps. It was shown that by controlling the $\mathrm{C}_{2} / \mathrm{H}_{\alpha}$ ratio it is possible to adjust the nucleation density and the growth rate of the deposited films (Woehrl and Buck 2011). These experiments lead to the idea, that the $\mathrm{C}_{2} / \mathrm{H}_{\alpha}$ ratio can also be used to adjust the deposition process between a preferential graphene and a preferential nanodiamond growth on copper substrates.

\section{Diagnostics}

To monitor the plasma properties the optical emission spectrometer (OES) was coupled to the process chamber via a glass fiber. The entire system was calibrated with a tungsten strip lamp. The influence of the process parameters on the carbon dimer $\mathrm{d} 3 \Pi \rightarrow \mathrm{a} 3 \Pi$ Swan band emission at $516 \mathrm{~nm}$ was investigated and a correlation with the film properties (quality of the graphene, nucleation density of diamond, growth rate of diamond) was made. A linear correlation between the intensity of the $C_{2}$-line and the actual $C_{2}$ concentration in the plasma has been proved (Goyette et al. 1998) and therefore the measurement of a $\mathrm{C}_{2} / \mathrm{H}_{\alpha}$-ratio provides quantitative information about the plasma and thus can be considered to be a reasonable measure for the plasma chemistry.

Raman spectroscopy is used for the characterization of graphene because it is a popular non-destructive analytical tool used to characterize the structure of the carbon materials (Malard et al. 2009). Raman spectroscopy is also suitable to determine the number of graphene layers, the disorder and the doping of the graphene (Ferrari et al. 2006; Casiraghi et al. 2007). The Raman spectra in this paper were measured with a Renishaw inVia REFLEX Raman spectrometer with a $532 \mathrm{~nm}(2.34 \mathrm{eV})$ and a $633 \mathrm{~nm}(1.96 \mathrm{eV})$ laser.

The Raman spectrum of graphene has a few prominent features. The G-band at around $1580 \mathrm{~cm}^{-1}$ arises from the $E_{2 g}$ in-plane vibration of $\mathrm{sp}^{2}$ carbon atoms. Two additional features, the $\mathrm{D}$-band which is assigned to the $\mathrm{A}_{1 \mathrm{~g}}$ breathing mode at the Brillouin Zone boundary $\mathrm{K}$ and the $\mathrm{D}^{\prime}$-band appear at around 1330 and $1620 \mathrm{~cm}^{-1}$, respectively, when point defects are introduced in the graphene layer as demonstrated in a controlled 
manner by ion implantation (Dresselhaus et al. 2010). Therefore $\mathrm{I}_{\mathrm{D}} / \mathrm{I}_{\mathrm{G}}$ intensity ratio can be used to quantify disorder in a graphene monolayer by determining the average distance between defects $L_{D}$ (Lucchese et al. 2010). Moreover, for large disorder the full width at half-maximum (FWHM) of the D and the G-band is even a better measure for structural disorder (Cançado et al. 2011).

The D $+\mathrm{D}^{\prime}$ band (around $2920 \mathrm{~cm}^{-1}$ ) is the combination of phonons with different momenta around $\mathrm{K}$ and $\Gamma$ and therefore provides a measure for the defect density.

The 2D band is the second order of the D band (Tuinstra and Koenig 1970; Gupta et al. 2006). Since the $2 \mathrm{D}$ band originates from the momentum conservation of two phonons with opposite wave vectors, no defects are required for its activation. The shape of the $2 \mathrm{D}$ band is of special interest because it provides information about the number of graphene layers in the material. In the case of single-layer graphene, the 2D band is fitted by a single narrow Lorentzian function. A FWHM of $33 \mathrm{~cm}^{-1}$ (measured with $532 \mathrm{~nm}$ ) for the 2D band is typically assigned to defect free SLG (Stampfer et al. 2007), whereas for few-layer samples the 2D feature is becoming significantly broader and asymmetric. Bilayer graphene has a much broader and up-shifted $2 \mathrm{D}$ band with respect to single-layer graphene due to its special electronic structure, consisting of two conduction bands and two valence bands (Malard et al. 2009; Ferrari et al. 2006; Jorio et al. 2010). Additionally, the intensity of the $\mathrm{G}$ band increases with increasing layer thickness, which allows further estimation of the layer thickness (Wang et al. 2008; Ni et al. 2010).

The UNCD crystals on the surface can also be characterized by Raman spectroscopy. The main features found in the spectra of UNCD are the already described Dand G-peak due to $\mathrm{sp}^{2}$-bonded carbon in the film. Two additional peaks at 1150 and $1480 \mathrm{~cm}^{-1}$ are assigned to NCD and UNCD although their true origin is still discussed. Some authors found evidence that these peaks originate from trans-polyacetylene in the grain boundaries (Castiglioni et al. 2004; Ferrari and Robertson 2004; Birrell et al. 2005). Polyacetylene is an organic polymer with the repeat unit $\left(\mathrm{C}_{2} \mathrm{H}_{2}\right)_{\mathrm{n}}$. The polymer consists of a chain of carbon atoms with alternating single and double bonds between them, each bonded with one hydrogen atom. In this paper Raman spectroscopy is used to quantitatively identify UNCD.

Additionally SEM measurements (JSM-7500F, JEOL) are used in this work to identify the crystalline structure of the deposited diamond crystals as well as to measure the nucleation density and the growth rate of the diamond crystals.

\section{Results and discussion}

Influence of plasma parameters on substrate temperature

OES is conducted to outline the influence of the macroscopic parameters pressure and methane concentration on the $\mathrm{C}_{2} / \mathrm{H}_{\alpha}$-ratio in the plasma at constant microwave power $1 \mathrm{~kW}$. In Fig. 2 the evolution of the $\mathrm{C}_{2} / \mathrm{H}_{\alpha}$-ratio is depicted as a function of the pressure in a range from 20 to 80 mbar. In addition to that, the methane concentration in the plasma is alternated from 5 to $30 \%$ in steps of $5 \%$. The measured values are shown as black spots whereas the grey levelled area is a 3D logistic fit. Both increasing pressure and increasing methane concentration lead to a strictly monotonically increasing $\mathrm{C}_{2} / \mathrm{H}_{\alpha}$-ratio. 


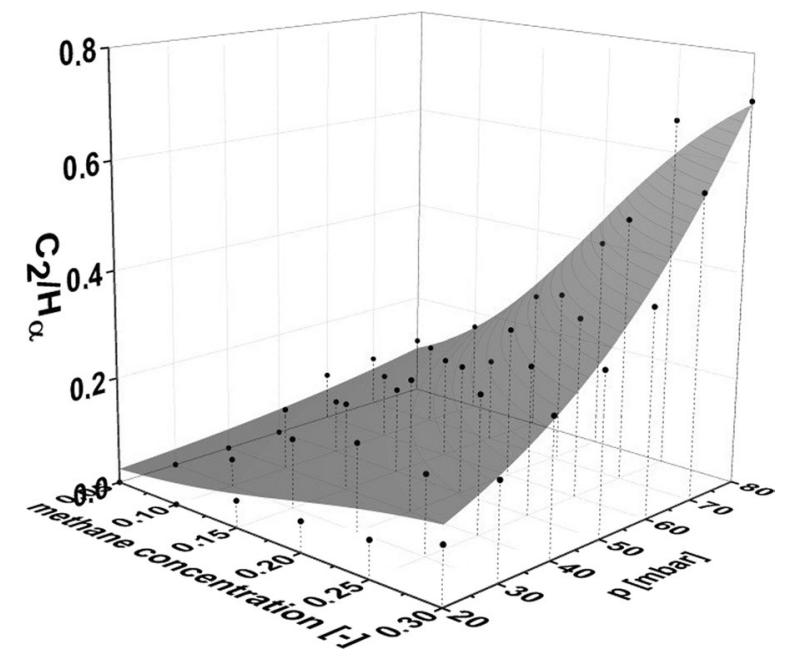

Fig. 2 Plasma chemistry pictured in a 3D plot. 3D area showing the behavior of the $C_{2} / \mathrm{Ha}$-ratio as a function of the applied pressure and the methane ratio in the gas mixture. The microwave power is held at a constant value of $1 \mathrm{~kW}$ for all measuring points depicted. The bullet points are the measured values for pressures between 20 and 80 mbar and methane ratios in the gas of between 5 and $30 \%$ respectively. The grey leveled area is a logistic fit of the total amount of measured values

As for the deposition of graphene on copper the substrate temperature has been found to have an important influence on the quality of the graphene, temperature measurements of the substrate are conducted as a function of process pressure. The substrate temperature is determined using an optical pyrometer. The general trend in this diagram is that higher pressure leads to higher substrate temperatures that originate form a denser plasma and thus more high-energetic particles interacting with the substrate. However, the two parts of the diagram shown in Fig. 3 separated by the red dashed line are found to depend on two different power laws. For small pressures the dependency is as follows

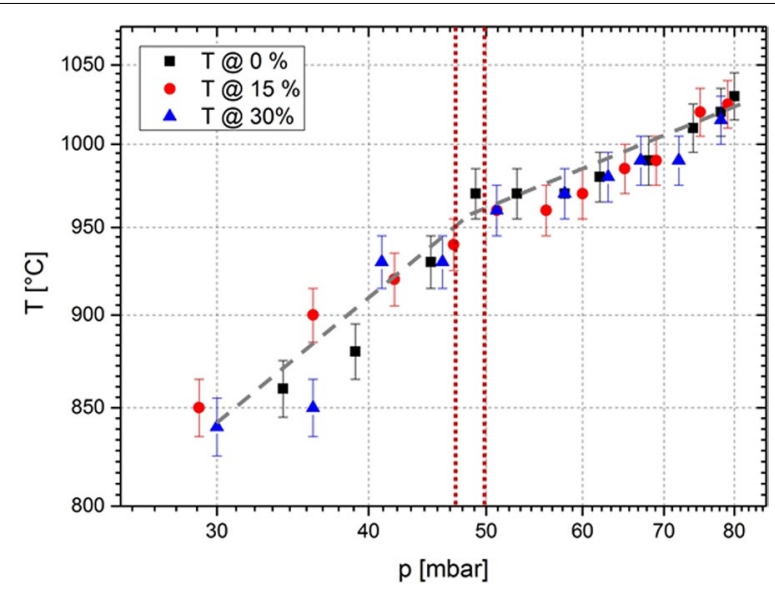

Fig. 3 Substrate temperature as a function of the pressure at different methane concentrations. The substrate temperature for different methane concentrations is plotted as a function of the process pressure two different power laws can be identified by fitting the two regimes in a log/log-plot. The vertical dashed lines indicate the approximate transition region between the two regimes 


$$
T \propto p^{2.5}
$$

whereas for high pressures the temperature as a function of the pressure can be described as

$$
T \propto p^{0.17}
$$

The change in slope originates from different heating mechanisms. The higher process pressure also leads to a reduced plasma ball size. As for the higher pressure above approx. 50 mbar the plasma ball is found to lose contact to the substrate beneath. Although the substrate is still heated by the radiation originating from the plasma, direct heat transfer by energetic particles is strongly reduced. This is because the distance between the plasma and the substrate is in the range of $\mathrm{mm}$ and energetic particles lose their energy by collisions in the gas, since the mean free path of molecules at a pressure of several tens of mbar is only in the range of a few $\mu \mathrm{m}$.

Repeating the measurements for different methane concentrations (0, 15 and $30 \%)$ reveals that there is no significant influence of the methane concentration on the pressure-temperature-dependency.

\section{Influence of the $\mathrm{C}_{2} / \mathrm{H}_{\mathrm{a}}$-ratio}

As the size of the plasma ball in MW PE-CVD depends on both the process pressure and the microwave power, these two macroscopic parameters influence the substrate-plasma coupling and therefore the substrate temperature (Rau and Trafford 1990). In order to investigate the influence of the $\mathrm{C}_{2} / \mathrm{H}_{\alpha}$-ratio on the quality of graphene properly, both substrate-plasma coupling and substrate temperature need to be kept constant.

Therefore experiments are conducted at constant microwave power and constant pressure using different methane concentrations in the process gas. In order to obtain a reasonable substrate temperature for the deposition of graphene on copper, $50 \mathrm{mbar}$ and $1 \mathrm{~kW}$ microwave power are chosen. They correspond to a substrate temperature of $960{ }^{\circ} \mathrm{C}$ (for an overview of detailed process parameter see Table 2). A temperature in this range is considered to be reasonable for the deposition of graphene in a copper-catalyzed CVD process ( $\mathrm{Li}$ et al. 2009; Srivastava et al. 2010). Since the methane concentration neither influences the substrate temperature, nor the plasma ball size significantly, the desired change of the plasma chemistry is triggered by increasing methane concentrations. Methane concentrations of 1,14 and $27 \%$ lead to $\mathrm{C}_{2} / \mathrm{H}_{\alpha}$-ratios of $0.045,0.14$ and

Table 2 Process parameters applied for the experiments investigating the $C_{2} / H_{a}$-ratio influence

\begin{tabular}{llll}
\hline $\mathrm{T}\left({ }^{\circ} \mathrm{C}\right)$ & 960 & 960 & 960 \\
$\mathrm{C}_{2} / \mathrm{H}_{\mathrm{a}}$ & 0.3 & 0.14 & 0.045 \\
$\mathrm{P}(\mathrm{mbar})$ & 50 & 50 & 50 \\
$\mathrm{P}(\mathrm{kW})$ & 1 & 1 & 1 \\
Methane concentration & 0.27 & 0.14 & 0.01 \\
\hline
\end{tabular}

In italic letters the desired $\mathrm{C}_{2} / \mathrm{H}_{\mathrm{a}}$-ratio is depicted. Using the information provided by the Figs. 2 and 3, sets of parameters can be found that facilitate the changes $\mathrm{C}_{2} / \mathrm{H}_{\mathrm{a}}$-ratio while the plasma ball dimensions and the substrate-plasma-interaction remains unchanged 
0.3 respectively. The experiments are conducted providing a total gas flux of $120 \mathrm{sccm}$ and a deposition time of $8 \mathrm{~min}$.

The samples are investigated with respect to the quality of the deposited graphene film. Overview photos reveal that during the process black spots are deposited. It is subsequently shown by Raman spectroscopy and SEM images that these black spots are almost ball-shaped nanodiamond clusters. In Fig. 4 photos of the surfaces are presented where the $\mathrm{C}_{2} / \mathrm{H}_{\alpha}$-ratio decreases from the top photo $\left(\mathrm{C}_{2} / \mathrm{H}_{\alpha}=0.3\right)$ to the bottom $\left(\mathrm{C}_{2} /\right.$ $\left.\mathrm{H}_{\alpha}=0.045\right)$. All the photos are taken with a $100 \times$ magnification. An SEM image of the diamond crystals is shown in Fig. 5. Small facets on the front indicate the crystalline structure of the spheres.
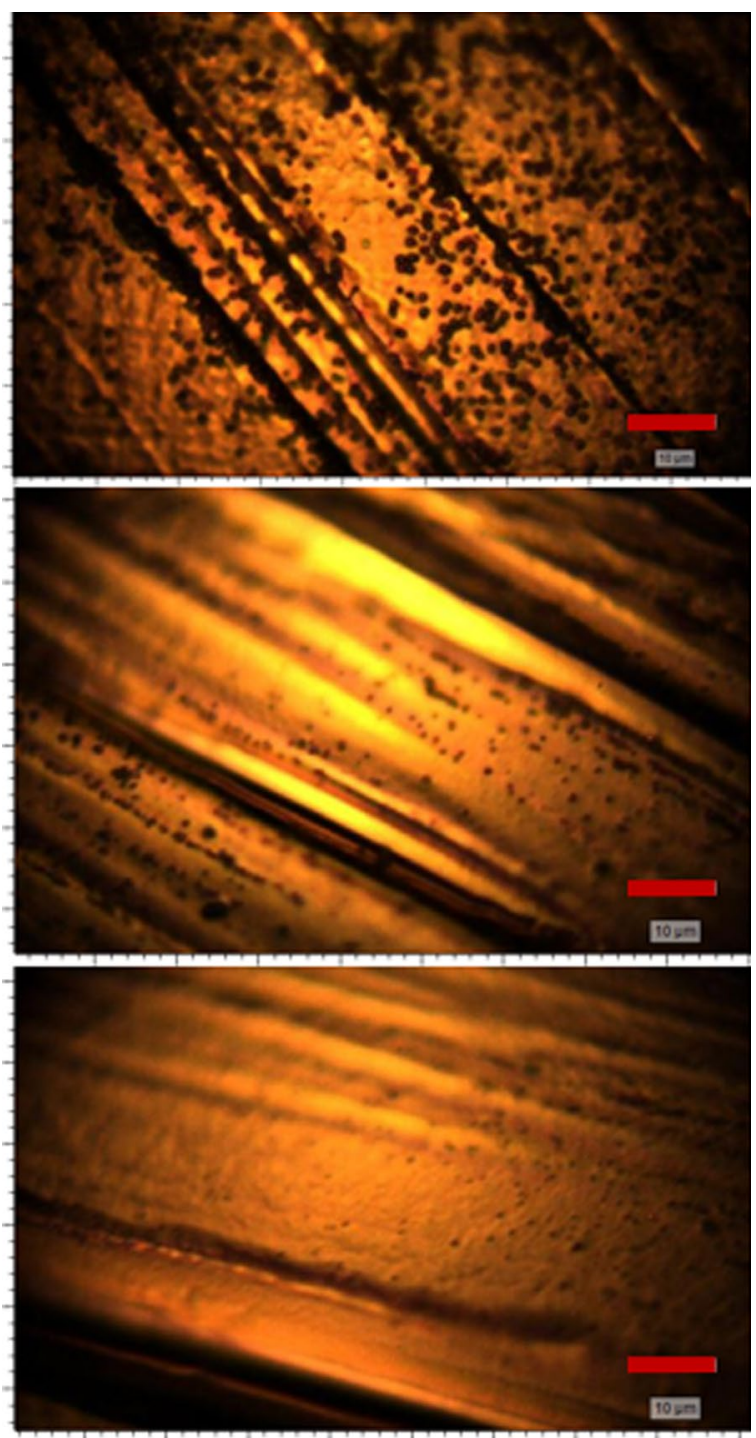

Fig. 4 Overview photos of samples synthesized under different plasma conditions. Left $\mathrm{C}_{2} / \mathrm{H}_{a}=0.3$, middle $\mathrm{C}_{2} / \mathrm{H}_{a}=0.14$ and right $\mathrm{C}_{2} / \mathrm{H}_{a}=0.045$ realized by reducing the methane concentration. Photos are taken by an optical microscope using a $\times 100$ magnification. All scale bars indicate a length of $10 \mu \mathrm{m}$. While the size of the nanocrystalline diamond spheres is proportional to the $\mathrm{C}_{2} / \mathrm{H}_{\mathrm{a}}$-ratio, a higher nucleation rate can be observed as the $\mathrm{C}_{2} / \mathrm{H}_{\mathrm{a}}$-ratio is reduced 


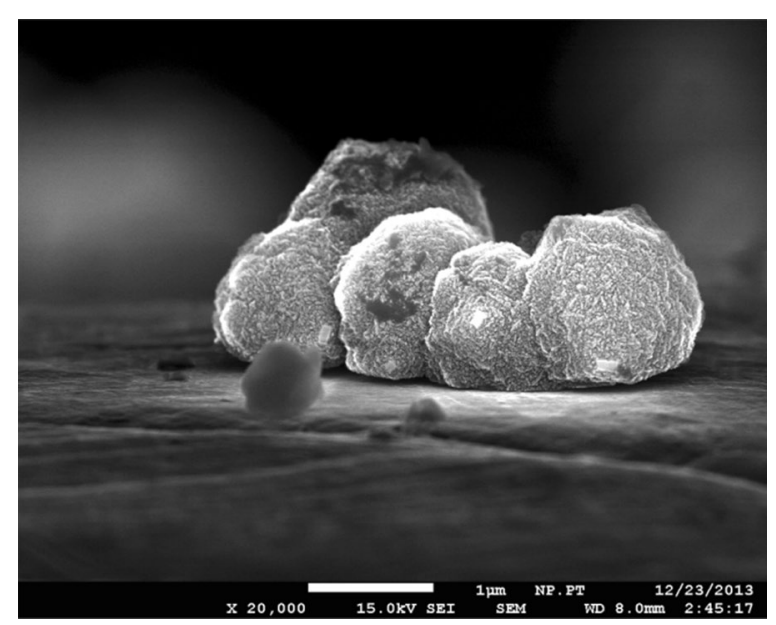

Fig. 5 High resolution SEM image of diamond spheres. The image shows a typical accumulation of nanodiamond spheres on a graphene-covered copper surface, the length of the scale bar is $1 \mu \mathrm{m}$. Diamond facets can be observed on the front side of the second sphere from the right

Graphene is found in the areas between the nanodiamond clusters. Figure 6 shows one representative Raman spectrum for every sample of the area between the spots. For all Raman spectra in this chapter a background subtraction is applied. From the analysis of the Raman spectra it can be derived that the $\mathrm{C}_{2} / \mathrm{H}_{\alpha}$-ratio has no decisive influence on the quality of graphene on copper substrates. On all the samples a constant $\mathrm{I}_{2 \mathrm{D}} / \mathrm{I}_{\mathrm{G}}$-ratio can be found. Moreover the $\mathrm{I}_{\mathrm{D}} / \mathrm{I}_{\mathrm{G}}$-ratio is constant for all the investigated samples. The symmetric shape of the 2D-peaks is commonly considered to be a hint for monolayer graphene. However, the high intensity of the D-Peak and the shoulder of the G-Peak are strong evidences for graphene structures with high defect density. Quantitatively the slight broadening of the three characteristic peaks in combination with an $\mathrm{I}_{\mathrm{D}} / \mathrm{I}_{\mathrm{G}}$-ratio of

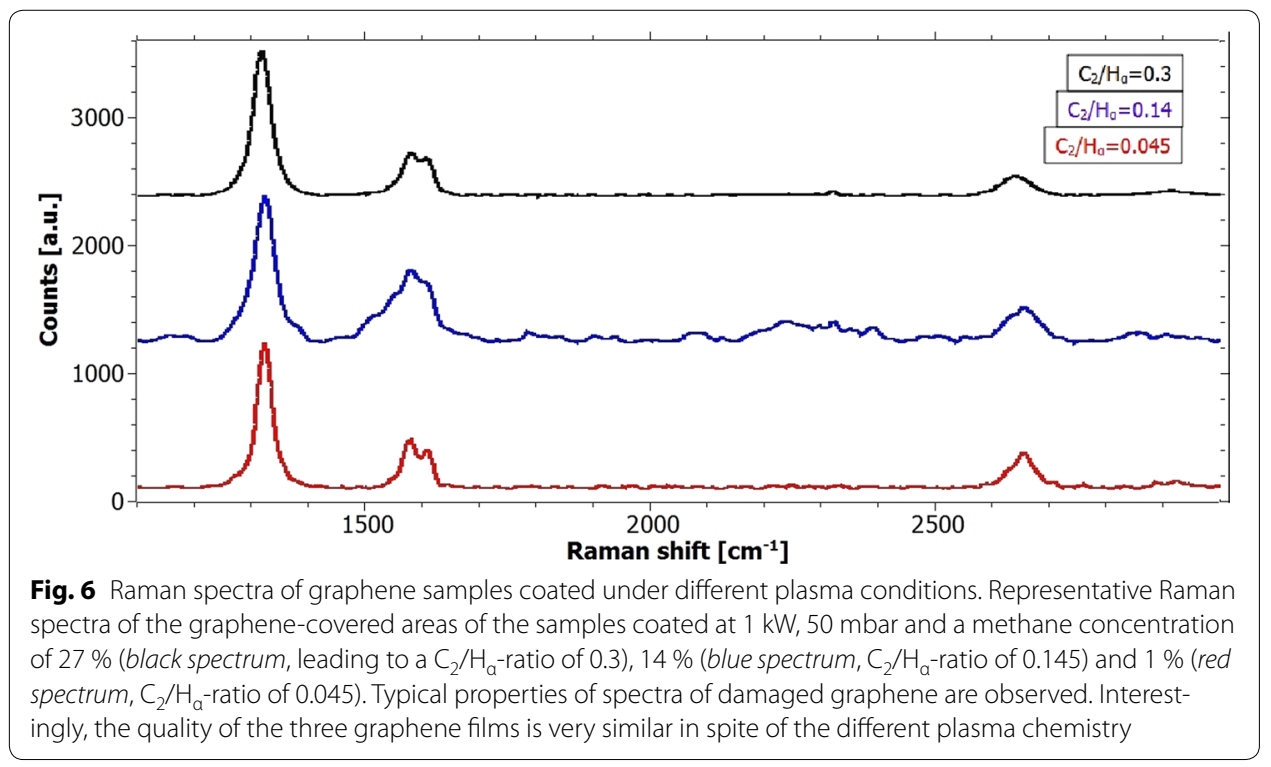


about three is indicating an average distance between point defects of about $2 \mathrm{~nm}$ in the measured graphene layers (Cançado et al. 2011).

In contrast to the widely unchanged graphene quality, the Raman spectra of the nanocrystalline diamond clusters seem to reveal a notable structural difference between the three samples (see Fig. 7). While the previous Raman spectra suggest the deposition of exclusively $\mathrm{sp}^{2}$-bonded carbon, the spectra of the diamond clusters taken between 1000 and $1800 \mathrm{~cm}^{-1}$ reveal the ordninary shape of nanocrystalline diamond films. The Raman spectra of all diamond clusters reveal the four most significant features of nanocrystalline diamond, namely the two broad peaks around 1335 and $1560 \mathrm{~cm}^{-1}$ (D- and G-Peak respectively) and the two humps located at about 1150 and $1480 \mathrm{~cm}^{-1}$ (Sharma et al. 2011). D and G peaks are both due to $\mathrm{sp}^{2}$ bonded carbon situated at the grain boundaries in NCD films. Since all spectra were taken by lasers in the visible light range only larger diamond crystals would feature a sharp line at $1332 \mathrm{~cm}^{-1}$ in the spectra originating from the $\mathrm{sp}^{3}$ bonded carbon. The results obtained from this analysis indicate that the spheres on the surface are in fact agglomerations of small nanodiamond crystals with sizes below $100 \mathrm{~nm}$.

Further interpretation of the nanodiamond spheres is difficult with Raman spectroscopy because the laser spot size is notably larger than the spheres that are meant to be investigated. Consequently a high percentage of the signal originates from interactions of the laser spot with surrounding and underlying graphene.

The volume $\mathrm{V}$ of the diamond clusters and the nucleation density of theses clusters $\rho_{\mathrm{A}}$ on the copper substrate was measured as a function of the $\mathrm{C}_{2} / \mathrm{H}_{\alpha}$-ratio (Fig. 8). It is shown that the average sphere volume increases for higher $\mathrm{C}_{2} / \mathrm{H}_{\alpha}$-ratios. In contrast to that the nucleation density is smaller for higher $\mathrm{C}_{2} / \mathrm{H}_{\alpha}$-ratios. Based on these results it can be stated that the nucleation of diamonds on the copper surface takes place in plasmas with lower carbon content whereas the nucleation of diamond on diamond (hence the growth of the diamond clusters) requires possibly high $\mathrm{C}_{2}$ ratios.

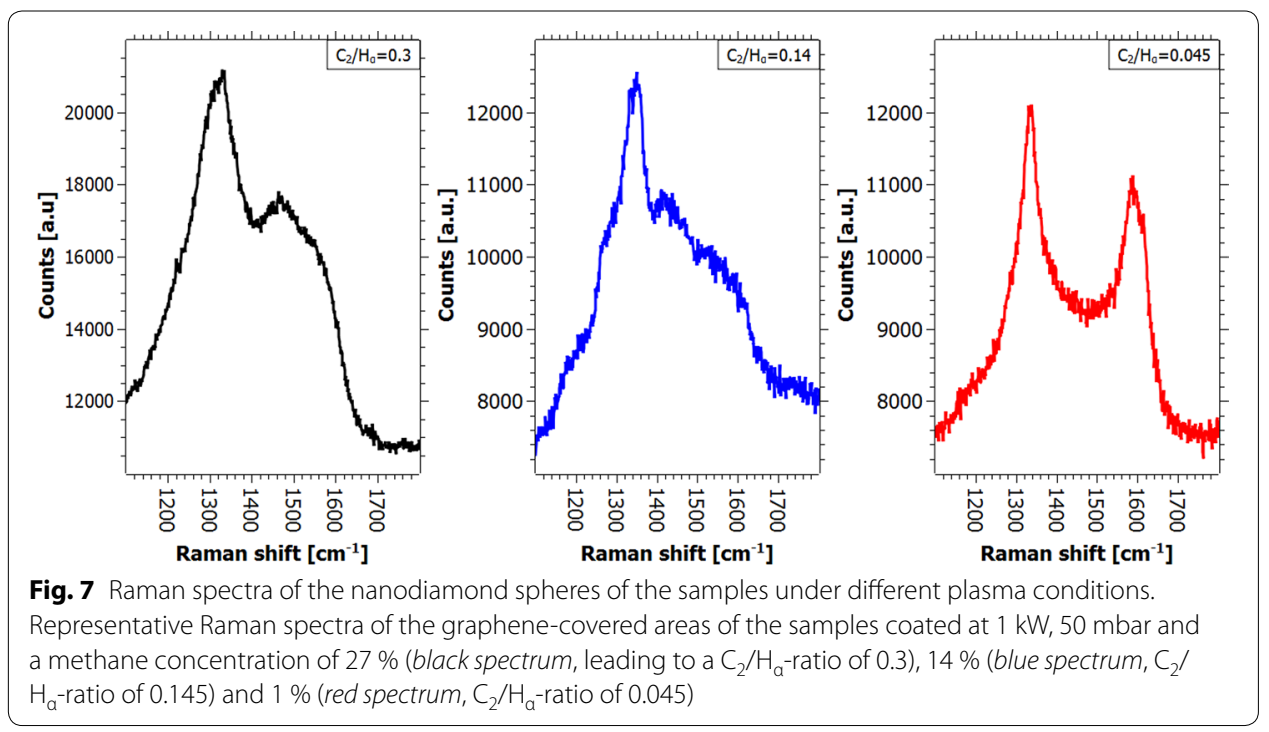


The overall deposition efficiency of $\mathrm{sp}^{3}$-bonded carbon is estimated as the product of the volume of one sphere and their area density divided by the concentration of methane in the gas flux (see last row of the table in Fig. 8). A small coefficient indicates inefficient deposition whereas high deposition efficiencies are characterized by large coefficients. For the three experiments conducted within this series it can be concluded that the deposition efficiency of $\mathrm{sp}^{3}$-bonded carbon is much higher for small a methane content and thus a respectively small $\mathrm{C}_{2} / \mathrm{H}_{\alpha}$-ratio. The observation of improved diamond growth in plasmas with a poor methane concentration is in good agreement with the literature (Woehrl and Buck 2007; Sharma et al. 2010).

Summarizing the results of this paragraph, the quality of the synthesized graphene does not significantly depend on the $\mathrm{C}_{2} / \mathrm{H}_{\alpha}$-ratio measured in the plasma. The growth of nanodiamonds (nucleation density and diamond cluster size) which occurs in all the experiments, however, can be strongly influenced by the $\mathrm{C}_{2} / \mathrm{H}_{\alpha}$-ratio. This finding suggests that the $\mathrm{C}_{2} / \mathrm{H}_{\alpha}$-ratio is a process parameter that allows it to influence the growth of the different carbon allotropes independently. Moreover, the results confirm that the chemical processes that lead to nucleation and growth of graphene and diamond on copper are significantly different and that specific process parameters can be used to synthesize hybrid structures.

\section{Influence of the plasma-ball size}

Since the interaction of the substrate surface with the energetic particles from the plasma (ions, electrons) is believed to lead to point defects in the synthesized graphene layers another experiment has been conducted with a larger distance between the substrate and the plasma to reduce this influence. Therefore, the microwave power is chosen to be $1 \mathrm{~kW}$ at a pressure of 65 mbar leading to a substrate temperature of about $990{ }^{\circ} \mathrm{C}$ which is close to the previous process temperatures. The methane concentration was chosen to be as low as $1 \%$ providing a $\mathrm{C}_{2} / \mathrm{H}_{\alpha}$-ratio of merely 0.02 . Although based on the results obtained from the previous experiments, an elevated nucleation density on the surface is expected, the nanodiamond particle growth is supposed to be widely inhibited. The results are presented in Fig. 9.

\begin{tabular}{|c|c|c|c|}
\hline $\mathbf{C}_{2} / \mathbf{H}_{\alpha}$ & 0.045 & 0.14 & 0.3 \\
\hline $\begin{array}{c}\mathrm{V} \\
{\left[\mu \mathrm{m}^{3}\right]}\end{array}$ & 0.112 & 0.291 & 1.142 \\
\hline $\begin{array}{c}\rho_{\mathrm{A}} \\
{\left[1 / \mu \mathrm{m}^{2}\right]}\end{array}$ & 0.92 & 0.52 & 0.28 \\
\hline$\frac{\mathrm{V} * \rho_{\mathrm{A}}}{c}$ & 10.34 & 1.08 & 1.184 \\
\hline
\end{tabular}
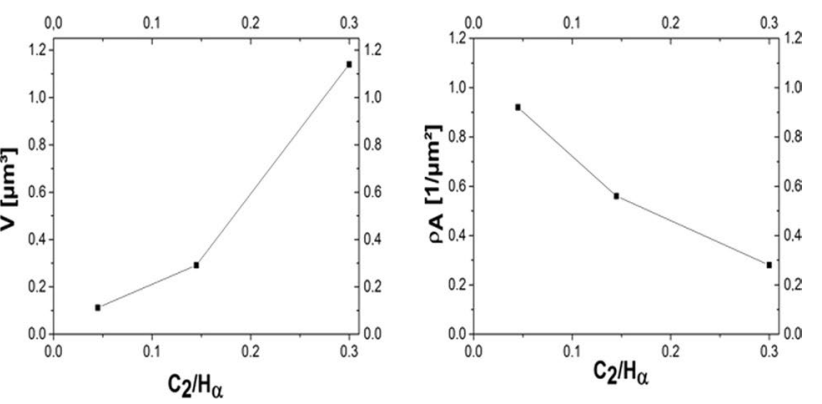

Fig. 8 Area density and size analysis of nanodiamond spheres. Results of analysis for the sphere size and the sphere area density for increasing methane concentrations (left) and the graphical result for sphere size (middle) and the sphere's area density (right) as a function of the $\mathrm{C}_{2} / \mathrm{H}_{\mathrm{a}}$-ratio. An increasing $\mathrm{C}_{2} / \mathrm{H}_{\mathrm{a}}$-ratio leads to taller nanodiamond spheres whereas the $\mathrm{C}_{2} / \mathrm{H}_{\mathrm{a}}$-ratio is inversely proportional to the number of spheres per unit area 


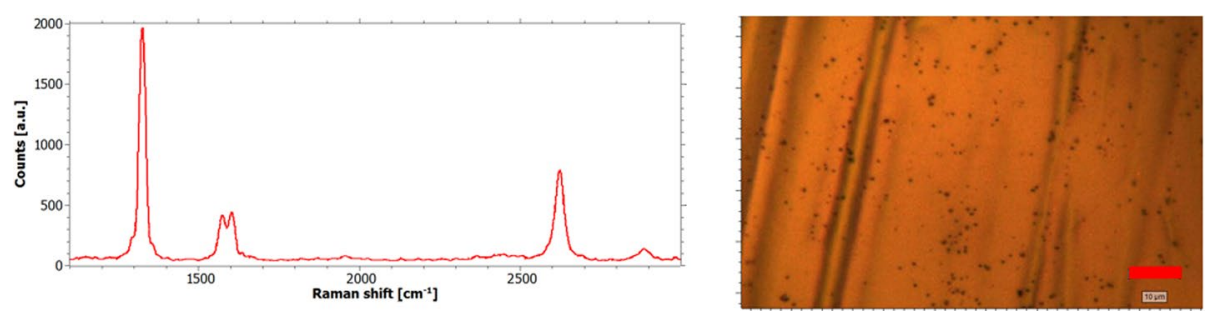

Fig. 9 Results obtained from reduced substrate-plasma-interaction. The application of a higher process pressure ( $65 \mathrm{mbar}$ instead of $50 \mathrm{mbar}$ ) leads to a compressed plasma ball and therefore reduces the plasmasubstrate-interaction. Left Overview of surface of sample. The size of the nanodiamond spheres could be further diminished. Right A characteristic Raman spectrum reveals an improved film quality with respect to the defect density

The results ought to be compared to the sample depicted in red in Fig. 6 (50 mbar, $1 \mathrm{~kW}$ and $1 \%$ methane) which was synthesized at similar conditions but in direct contact with the plasma. It is found that both nucleation and growth of the nanodiamonds is reduced by enlarging the distance between the substrate and the plasma. The Raman spectrum of the sample reveals a considerably better result than the previous samples with respect to the graphene quality. From the narrow peaks and the $I_{D} / I_{G}$-ratio of approximately 4.5 , a distance of $7 \mathrm{~nm}$ between two point defects can be derived. Therefore the average distance of point defects could be reduced by a factor of 5.3 by the larger distance between plasma and substrate.

Detailed peak analysis shows that monolayer graphene has been deposited. As there is no peak shift detected (G-peak at $1586 \mathrm{~cm}^{-1}, \mathrm{D}^{\prime}$-peak at $1618 \mathrm{~cm}^{-1}$ and 2D-peak at $2661 \mathrm{~cm}^{-1}$; spectrum taken at $1.96 \mathrm{eV}$ laser excitation energy) there is neither considerable strain applied to the sheet, nor sheet doping can be reported.

\section{Influence of the direct plasma contact}

It has been shown that the contact of the sample to the plasma has a negative influence on the quality of the deposited graphene. In contrast to that the direct plasma contact is found to be advantageous in order to synthesize nanodiamonds. This result is in good agreement with earlier experiments explaining the nucleation of diamond by charged or polar precursors in the plasma (Buck 2008).

In order to obtain additional information about the different growth mechanisms of diamond and graphene and the role the direct plasma contact has on these mechanisms a sample with a substrate geometry as shown in Fig. 10a has been fabricated.

A cavity is formed in order to enable diffusive transport to the back side of the sample, while inhibiting a direct contact to the plasma. In contrast, the upper side of the sample has direct plasma contact. Equal temperatures at the front side and at the back side of the sample are obtained in this constellation and thus the only difference between the two sides is the plasma-sample interaction.

The sample is processed at $50 \mathrm{mbar}, 1 \mathrm{~kW}$ and a methane concentration of $27 \%$ resulting in a substrate temperature of $960{ }^{\circ} \mathrm{C}$ and a $\mathrm{C}_{2} / \mathrm{H}_{\alpha}$-ratio of 0.3 . After the deposition the two sides of the sample are investigated separately (results see Fig. 10b-e).

Whilst strong diamond nucleation is observed at the front of the substrate, no nanodiamond can be found at its back side (see overview photos in Fig. 10b/c). Interestingly 


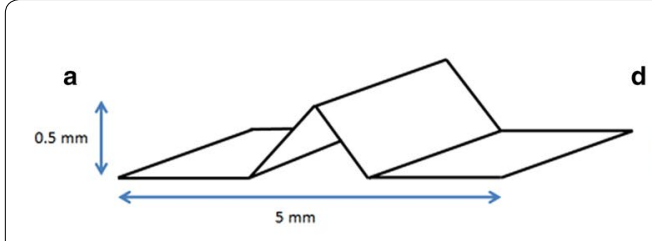

b

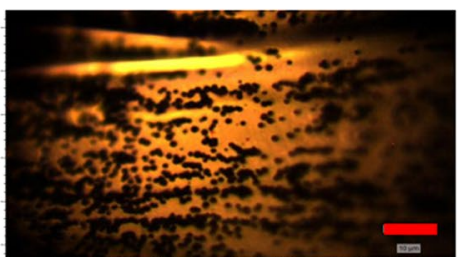

e

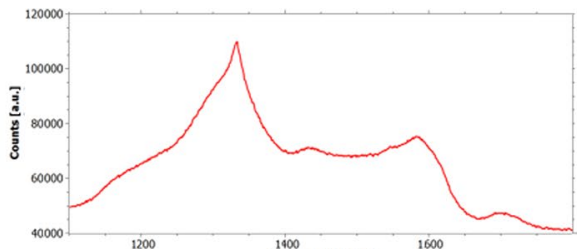

䨔

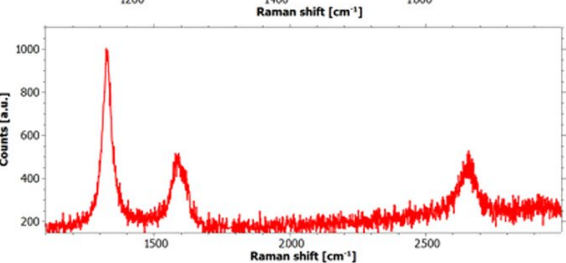

c

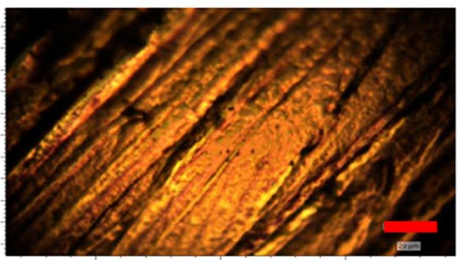

$\mathbf{f}$

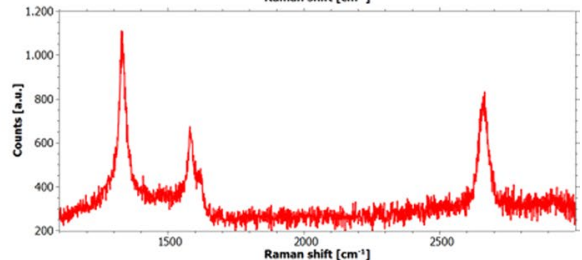

Fig. 10 Overview of the influence of direct plasma contact. a The triangular shaped sample enables diffusive transport of species into the cavity whereas the upper side of the surface experiences a direct plasma contact. $\mathbf{b}$ Overview photo of the upper side of the sample: Massive nanodiamond nucleation has taken place as a result from the substrate-plasma-interaction. The length of the scale bar is $10 \mu \mathrm{m}$. c Overview photo of the substrate surface in the cavity. No nanodiamond nucleation is observed. Length of scale bar: $20 \mu \mathrm{m}$. d Raman spectrum of nanodiamond spheres on the front side. Characteristic diamond peaks are visible. e Raman spectrum of graphene between nanodiamond spheres: damaged graphene has been synthesized. f Raman spectrum of graphene in the cavity on the back side of the sample. Damaged graphene of a similar quality to the front side is observed. The lack of plasma interaction leads to a slightly improved result compared to the front side

the defect density in the graphene sheet at the front side and the back side is similar. In both cases the mean distance between two point defects is estimated to be $3-4 \mathrm{~nm}$. This can lead to the conclusion that most of the point defects in the graphene are not due to plasma surface interactions but due to the high temperature of the copper and the resulting evaporation during the growth.

The most important outcome, however, is that evidence of the different growth mechanisms of diamond and graphene is shown. Direct plasma contact is inevitable for the growth of diamonds. In contrast to that the growth of graphene is based on the temperature activated catalytic influence of copper which does not require direct plasma contact.

\section{Conclusions}

In this paper the simultaneous growth of nanodiamond clusters and graphene on copper by PE-CVD process is described for the first time. It is found that the $\mathrm{C}_{2} / \mathrm{H}_{\alpha}$-ratio in the plasma is a process parameter that allows to influence the growth of the different carbon allotropes independently. While the diamond nucleation can be influenced by the $\mathrm{C}_{2} /$ $\mathrm{H}_{\alpha}$-ratio, the graphene quality remains mostly unchanged by this parameter. Moreover, it is derived from the experimental data that the direct plasma contact with the copper surface is beneficial for the nucleation of the diamond clusters while the growth and 
quality of the graphene benefit from a larger distance to the plasma. Therefore, this work identifies crucial parameters to establish a method to deposit graphene-diamond hybrid films using a MW PE-CVD process, tailor the composition of these films, or entirely oppress the diamond deposition if desired.

\section{Abbreviations}

MW PE-CVD: microwave plasma enhanced chemical vapor deposition; OES: optical emission spectroscopy; SLG: single layer graphene; NCD: nanocrystalline diamond; UNCD: ultra nanocrystalline diamond; FWHM: full width half maximum.

\section{Authors' contributions}

SG carried out all the experiments presented in this work. NW conceived the experimental studies and participated in the evaluation of the experimental data. VB participated in the coordination of the project and helped to draft the manuscript. SS also helped to draft the manuscript. All authors read and approved the final manuscript.

\section{Authors' information}

SG is a master student of the NanoEngineering study program at the University of Duisburg-Essen. NW holds a doctorate degree in physics and is currently "Plasma treatment" group leader in the Nano Energy Technique Center, Duisburg, Germany. VB and SS are professors in the faculties of physics and chemistry of the University of Duisburg-Essen, Germany.

\section{Author details}

${ }^{1}$ Faculty of Physics and CENIDE, University Duisburg Essen, Carl-Benz-Straße 199, 47057 Duisburg, Germany. ${ }^{2}$ Faculty of Chemistry and CENIDE, University Duisburg-Essen, Carl-Benz-Straße 199, 47057 Duisburg, Germany.

\section{Acknowledgements}

The authors would like to thank Sebastian Schipporeit (University Duisburg-Essen) for performing SEM studies on the nanodiamond clusters.

\section{Competing interests}

The authors declare that they have no competing interests.

Received: 13 November 2015 Accepted: 20 April 2016

Published online: 10 May 2016

\section{References}

Akturk A, Goldsman N (2008) Electron transport and full-band electron-phonon interactions in graphene. J Appl Phys 103(5):053702

Birrell J, Gerbi JE, Auciello O, Gibson JM, Johnson J, Carlisle A (2005) Interpretation of the Raman spectra of ultrananocrystalline diamond. Diam Relat Mater 14(1):86-92

Buck V (2008) Evidence for charged or polar precursor in diamond nucleation. J Optoelectron Adv Mater 10:85-90

Buck V, Woehrl N (2008) Tailoring the matrix in ultra-nanocrystalline diamond films. Jpn J Appl Phys 47:8208-8213

Cançado LG, Jorio A, Martins Ferreira EH, Stavale F, Achete CA, Capaz RM, Moutinho MVO, Lombardo A, Kulmala TS, Ferrari AC (2011) Quantifying defects in graphene via Raman spectroscopy at different excitation energies. Nano Lett 11:3190-3196

Casiraghi C, Pisana S, Novoselov KS, Geim AK, Ferrari AC (2007) Raman fingerprint of charged impurities in graphene. Appl Phys Lett 91:233108

Castiglioni C, Tommasini M, Zerbi G (2004) Raman spectroscopy of polyconjugated molecules and materials: confinement effect in one and two dimensions. Philos Trans R Soc Lond A 361:1824

Chan SH, Chen SH, Lin WT, Li MC, Lin YC, Kuo CC (2013) Low-temperature synthesis of graphene on Cu using plasmaassisted thermal chemical vapor deposition. Nanoscale Res Lett 8(1):285-289

CYRANNUS ${ }^{\circledR}$ I (2001) European Patent 0872164, USA Patent 6198224

Dresselhaus MS, Jorio A, Hofmann M, Dresselhaus G, Saito R (2010) Perspectives on carbon nanotubes and graphene Raman spectroscopy. Nano Lett 10:751

Ferrari AC, Robertson J (2004) Raman spectroscopy of amorphous, nanostructured, diamond-like carbon, and nanodiamond. Philos Trans R Soc Lond A 362:2477

Ferrari AC, Meyer JC, Scardaci V, Casiraghi C, Lazzeri M, Mauri F, Piscanec S, Jiang D, Novoselov KS, Roth S, Geim AK (2006) Raman spectrum of graphene and graphene layers. Phys Rev Lett 97:187401

Goyette AN, Lawler JE, Anderson LW, Gruen DM, McCauley TG, Zhou D, Krauss AR (1998) Swan band emission as a function of denisty. Plasma Sour Sci Technol 7:149

Gruen DM (1999) Nanocrystalline diamond films 1. Annu Rev Mater Sci 29:211-259

Gruen DM, Pan X, Krauss AR, Liuy A, Luo J, Foster CM (1994) Deposition and characterization of nanocrystalline diamond films. J Vac Sci Technol, A 12:1491

Gupta A, Chen G, Joshi P, Tadigadapa S, Eklund P (2006) Raman scattering from high-frequency phonons in supported n-graphene layer films. Nano Lett 6:2667

Jorio A, Dresselhaus M, Saito R, Dresselhaus G (2010) Raman spectroscopy in graphene related systems, 1st edn. Wiley, Weinheim 
Kalita G, Wakita K, Umeno M (2012) Low temperature growth of graphene film by microwave assisted surface wave plasma CVD for transparent electrode application. RSC Adv 8:2815-2820

Kim Y, Song W, Lee SY, Jeon C, Jung W, Kim M et al (2011a) Low-temperature synthesis of graphene on nickel foil by microwave plasma chemical vapor deposition. Appl Phys Lett 8:263106

Kim J, Ishihara M, Koga Y, Tsugawa K, Hasegawa M, lijima S (2011 b) Low-temperature synthesis of large-area graphenebased transparent conductive films using surface wave plasma chemical vapor deposition. Appl Phys Lett 8:091502

Li X, Cai W, An J, Kim S, Nah J, Yang D, Piner R, Velamakanni A, Jung I, Tutuc E, Banerjee SK, Colombo L, Ruoff RS (2009) Large-area synthesis of high-quality and uniform graphene films on copper foils. Science 324:1312-1314

Lucchese MM, Stavale F, Martins Ferreira EH, Vilani C, Moutinho MVO, Capaz RB, Achete CA, Jorio A (2010) Quantifying ion-induced defects and relaxation length in graphene. Carbon 48:1592

Malard L, Pimenta M, Dresselhaus G, Dresselhaus M (2009) Raman spectroscopy in graphene. Phys Rep 473:51

Ni Z, Wang Y, Yu T, Shen Z (2010) Raman spectroscopy and imaging of graphene. Nano Res 4:273-281

Novoselov KS, Geim AK, Morozov SV, Jiang D, Zhang Y, Dubonos SV et al (2004) Electric field effect in atomically thin carbon films. Science 306:666-669

Rau H, Trafford B (1990) A microwave plasma ball reactor-experiment and simulation. J Phys D Appl Phys 23:1637-1641

Reina A, Jia X, Ho J, Nezich D, Son H, Bulovic V et al (2009) Large area, few-layer graphene films on arbitrary substrates by chemical vapor deposition. Nano Lett 9(1):30-35

Sharma R, Woehrl N, Barhai PK, BuckV (2010) Nucleation density enhancement for nanocrystalline diamond films. JOAM 12:1915-1920

Sharma R, Woehrl N, Vrućinić M, Timpner M, Buck V, Barhai PK (2011) Effect of microwave power and C2 emission intensity on structural and surface properties of nanocrystalline diamond films. Thin Solid Films 519:7632-7637

Srivastava A, Galande C, Ci L, Song L, Rai C, Jariwala D, Kelly KF, Ajayan PM (2010) Novel liquid precursor-based facile synthesis of large-area continuous, single, and few-layer graphene films. Chem Mater 22:3457

Stampfer C, Molitor F, Graf D, Ensslin K, Jungen A, Hierold C, Wirtz L (2007) Raman imaging of doping domains in graphene on $\mathrm{SiO}_{2}$. Appl Phys Lett 91:241907

Tuinstra F, Koenig F (1970) Raman spectrum of graphite. J Chem Phys 53:1126

Vlassiouk I, Regmi M, Fulvio P, Dai S, Datskos P, Eres G et al (2011) Role of hydrogen in chemical vapor deposition growth of large single-crystal graphene. ACS Nano 5(7):6069-6076

Wang YY, Ni ZH, Shen ZX, Wang HM, Wu YH (2008) Raman spectroscopy of epitaxial graphene on a SiC substrate. Appl Phys Lett 92:043121

Wang SJ, Geng Y, Zheng QB, Kim JK (2010) Fabrication of highly conducting and transparent graphene films. Carbon 8:1815-1823

Woehrl N, Buck V (2007) Influence of hydrogen on the residual stress in nanocrystalline diamond films. Diam Relat Mater $16: 748-752$

Woehrl N, Buck V (2011) Process control of CVD deposition of nanocrystalline diamond films by plasma diagnostics. Z Phys Chem 225:1379-1391

Woehrl N, Ochedowski O, Gottlieb S, Shibasaki K, Schulz S (2014) Plasma-enhanced chemical vapor deposition of graphene on copper substrates. AlP Adv 4:047128

Zhang W, Wu P, Li Z, Yang J (2011) First-principles thermodynamics of graphene growth on Cu surface. J Phys Chem 115(36):17782-17787

\section{Submit your manuscript to a SpringerOpen ${ }^{\circ}$ journal and benefit from:}

- Convenient online submission

- Rigorous peer review

- Immediate publication on acceptance

Open access: articles freely available online

- High visibility within the field

- Retaining the copyright to your article

Submit your next manuscript at $>$ springeropen.com 